\title{
Les critères de choix de la spécialité des médecins résidents de la faculté de médecine et de pharmacie de Marrakech (Maroc)
}

\section{Factors influencing the career choice of resident physicians of the faculty of medicine and pharmacy of Marrakech (Morocco)}

\author{
Aboubakr MATRANE $^{1}$, Zineb SERHIER ${ }^{2}$ et Mohammed BENNANI OTHMANI ${ }^{2}$ \\ 1 Service de médecine nucléaire, Faculté de médecine et de pharmacie, Université Cadi Ayyad, Marrakech, Maroc \\ ${ }^{2}$ Laboratoire d'informatique médicale, Faculté de médecine et de pharmacie, Université Hassan II, Casablanca, Maroc
}

Manuscrit reçu le 31 juillet 2011; commentaires éditoriaux formulés aux auteurs le 13 novembre 2011 ; accepté pour publication le 11 juin 2012

\begin{abstract}
Mots-clés Étudiants en médecine ; facteurs ; choix de carrière

Résumé - Objectif : Étudier les facteurs qui influencent le choix de carrière des médecins résidants de la faculté de médecine et de pharmacie de Marrakech. Méthodes : Un questionnaire, adapté de celui déjà publié par Murdoch et al., composé de quatre parties a été distribué à 193 médecins résidents : la première partie concernait les caractéristiques sociodémographiques, la seconde partie le choix de spécialité choisie, la troisième partie correspondait aux facteurs personnels influençant le choix de la spécialité et la quatrième partie les caractéristiques attribuées aux choix des spécialités, réparties en six échelles de valeurs. Résultats : La chirurgie était un choix en très grande majorité masculin. La dermatologie et la gastroentérologie étaient des choix presque exclusivement féminins. Ces différences liées au sexe des médecins étaient aussi retrouvées au niveau du choix des échelles de valeurs. Les autres facteurs démographiques n'avaient pas d'influence notable sur le choix de spécialité. Les échelles de valeur étaient corrélées également avec les choix de spécialité des médecins. Ainsi, dans les échelles de valeurs « intérêt académique, prestige, rôle de soutien » plusieurs variables étaient liées au choix de la spécialité chirurgicale, tandis que dans l'échelle « évitement des contraintes » une variable était liée au choix de la spécialité médicale. Les échelles « orientation biosociale et bioscientifique » et « revenu » ne montraient aucune corrélation positive avec le choix de la spécialité des résidants. Conclusion : Le médecin est confronté au choix d'une spécialité et de sa future carrière professionnelle. L'attractivité pour certaines spécialités est très faible, liée certainement à des conditions d'exercice insuffisamment valorisées.
\end{abstract}




\section{Keywords}

Medical students;

factors; career choice
Abstract - Purpose: To study the factors influencing career choices among resident physicians at the faculty of medicine and pharmacy of Marrakech. Method: A four-part questionnaire was handed out to 193 resident physicians: the first part involved socio-demographic characteristics, the second part related to the chosen specialty, the third part concerned personal factors influencing the choice of the specialty and the fourth part covered the characteristics attributed to the selection of specialties, distributed in six value scales in accordance with Murdoch's study. Results: Surgery was selected overwhelmingly by male physicians while dermatology and gastroenterology were almost exclusively a female physician choice. The choice of value scales also reflected differences relating to physician gender. Other demographic factors had no significant influence on choice of specialty. Value scales were also correlated with the physician's choice of specialty. Accordingly, in "academic interest, prestige, role support" value scales, several variables were related to the choice of the surgical specialty, whereas in the "avoiding stress" scale, a single variable was related to the choice of a medical specialty. The "biosocial and bioscientific track" and "income" scales did not show any positive correlation with the specialty selected by residents. Conclusion: Physicians are confronted with choosing a specialty and their future professional career. Some specialties have very little appeal, probably due to the low value of practice conditions.

\section{Introduction}

La prise en charge optimale de la santé d'une population nécessite des soins de qualité, qui sont notamment liés à l'existence d'un dispositif adapté de soins primaires. Dans une telle perspective, la réforme du système de santé au Maroc réside dans l'accroissement de l'investissement en médecine générale, non seulement pour les ressources humaines et les infrastructures, mais aussi pour la formation, la recherche et la qualité de prise en charge des dépenses de soins de santé de base.

Alors qu'un certain nombre d'éléments semblent valoriser cette discipline, les étudiants en médecine préfèrent pourtant d'autres spécialités plus honorifiques, plus reconnues et/ou plus lucratives. L'accès au cycle d'études médicales spécialisées (résidanat) est ouvert, par voie de concours national, aux candidats remplissant les conditions prévues par la réglementation en vigueur. Les postes sont fixés par arrêté conjoint de l'autorité gouvernementale chargée de l'enseignement supérieur, du ministère de la santé publique et, le cas échéant, de l'autorité chargée de l'administration de la défense nationale pour les résidents militaires ${ }^{[1]}$. Après validation de la cinquième année des études de médecine, les étudiants, s'ils le veulent, peuvent passer le concours d'internat dans le centre hospitalier universitaire (CHU) de leur choix. Les internes admis effectuent quatre stages de six mois dans le CHU où ils ont été nommés. Après les deux années d'internat, les internes deviennent des résidents sur titre et choisissent la spécialité dans la liste des postes disponibles. Ces résidents sont tenus de soutenir leur thèse de doctorat d'exercice au plus tard durant la première année du résidanat. Les étudiants n'ayant pas passé le concours d'internat ou n'ayant pas été admis au concours, peuvent soutenir leur thèse pour l'obtention du doctorat d'exercice en médecine générale, une fois qu'ils ont validé les examens cliniques de la septième année. Après la soutenance, les médecins ont le droit de passer le concours de résidanat. Un classement définitif est établi après le concours, les futurs résidents choisissant par ordre de mérite leur future spécialité. En général, les résidents sur concours font leur choix, après les résidents sur titre, dans la liste des postes disponibles.

Avec l'objectif de combler le manque de médecins au Maroc, le ministère de la Santé a lancé un plan de santé très large destiné à former 3300 médecins par an à l'horizon 2020, pour atteindre une densité médicale en mesure d'assurer un encadrement médical correct de la population, projet qui est désormais possible grâce aux nouveaux CHU de Fès, Oujda et Marrakech $^{[2-4]}$. Afin de fournir aux décideurs des 
éléments fondés pour mettre en place un véritable plan et une politique de santé appropriés au paysage marocain en terme de programmation de concours de spécialité, il serait intéressant de connaitre les facteurs déterminant le choix de la spécialité par les médecins. Plusieurs études occidentales se sont intéressées à cette problématique, parmi lesquelles notamment celle de Murdoch et al. ${ }^{[5]}$, qui a identifié six échelles de valeur associées au choix de la spécialité. L'administration de ce questionnaire à des étudiants libanais avait permis de recueillir des résultats qui accréditaient sa validité de construit dans ce contexte en montrant des résultats similaires ${ }^{[6]}$. Le Maroc qui est également un pays méditerranéen a des caractéristiques sociodémographiques différentes de celle du Liban, notamment au regard de la grande taille de la population et du nombre insuffisant de médecins. L'objectif de cette étude était d'identifier les facteurs qui influencent le plus le choix de la spécialité des futurs médecins marocains spécialistes au cours de leur formation médicale.

\section{Matériel et méthodes}

Il s'agit d'une étude transversale réalisée en 2010. Elle a été menée auprès des résidents (sur titre et sur concours) de la Faculté de médecine et de pharmacie de l'Université Cadi Ayyad de Marrakech. Tous les résidents de la première à la cinquième année de résidanat ont été invités à participer à l'étude. La liste a été fournie par le service de scolarité de la faculté de médecine. Un questionnaire a été remis aux résidents et un temps de réponse libre leur a été ménagé.

Le questionnaire de trois pages comprenait 44 items groupés en quatre parties :

- une première partie de huit items recueillait des renseignements de nature socio-démographique (âge, sexe, année de résidanat, état civil, membre de famille professionnel de santé, niveau d'éducation des parents, activité de volontariat);

- une seconde partie de quatre items les invitait à énoncer leur choix de carrière (type de spécialité, type de résidanat, type de contrat, classement au concours) ;
- une troisième partie de 12 items recueillait les facteurs influençant leur choix de la spécialité (durée de formation, disponibilité du poste, affectation, qualité de la formation, défi, culte et tradition, influence de la famille ou du conjoint, profil personnel, choc émotionnel, responsabilité, nature de la spécialité) ;

- une quatrième partie de 20 items explorait des valeurs liées à la profession envisagée. Ces 20 items, extraits dans leur intégralité de l'étude princeps de Murdoch $^{[5]}$, sont répartis sur six échelles de valeurs (Annexe 1). Chacun de ces items est coté sur une échelle de Likert à cinq niveaux allant de «pas d'influence » à « influence très importante ».

Les données ont été saisies sur Excel et analysées par le logiciel SPPS 16.0. Les données qualitatives sont présentées sous forme de pourcentage. Les données quantitatives sont présentées sous forme de moyenne \pm écart type. Les réponses des médecins ont été analysées en fonction de leur statut et de leur spécialité.

Nous avons effectué une analyse descriptive simple des différentes variables étudiées, puis dans un second temps une analyse univariée par le test de Chicarré $\left(\chi^{2}\right)$, en exigeant le seuil de significativité de $5 \%$.

\section{Résultats}

\section{Description de la population d'étude}

Sur les 262 questionnaires distribués, 193 ontété récupérés, soit un taux de réponse de 73,7 \%. Environ $36,8 \%$ des participants étaient des résidents sur titre et $63,2 \%$ des résidents sur concours. Le meilleur pourcentage de participation $(94,3 \%)$ était enregistré en première année et le plus bas en cinquième année (56\%). Des commentaires libres complétaient 34 des 193 questionnaires $(17,6 \%)$. Les résidents étaient répartis respectivement en première année $(39,9 \%)$, en deuxième année $(24,4 \%)$, en troisième année $(17,1 \%)$, en quatrième année $(15,5 \%)$ et en cinquième année $(3,1 \%)$. La moyenne d'âge était de 29 ans (écart type $=3,4$ ). Le ratio hommes/femmes était de 81/112. 
Les deux sexes étaient représentés parmi les cinq promotions étudiées, à des proportions variées pour chaque promotion prise à part, avec des pourcentages de participation féminine de 71,4\%, 55,3\%, 55,8\%, $50 \%$ et $16,6 \%$ respectivement de la première à la cinquième année.

Le niveau d'instruction des parents était très variable. Le niveau primaire était plus fréquent chez les mères que chez les pères des médecins participant à l'enquête (28,7 \% à 32,1\% versus $12,2 \%$ à 14,1\%). Par contre, le niveau supérieur variait de $23,1 \%$ à $23,5 \%$ chez les mères et de $35,9 \%$ à $38,3 \%$ chez les pères.

\section{Type de carrière choisie et facteurs associés à ce choix}

Les carrières médicales étaient plus choisies par les étudiants et représentaient $59,6 \%$ des choix versus $40,4 \%$ pour les carrières chirurgicales dont $30,1 \%$ en gynécologie obstétrique, chirurgie viscérale, traumatologie et oto-rhino-laryngologie (ORL). Le choix des spécialités de pédiatrie, de néphrologie, de dermatologie et de cardiologie représentait $24,3 \%$ des carrières médicales (Figure 1).

Certaines carrières étaient respectivement plus fréquemment choisies par les hommes ou par les femmes. Ainsi, la pédiatrie a été choisie dans notre échantillon uniquement par les femmes $(11,7 \%$ des femmes ont choisi la pédiatrie). Les spécialités chirurgicales ont été choisies plus fréquemment par les hommes (68,4\% contre $21,1 \%$ de femmes), à l'exception de trois spécialités chirurgicales (la gynécologieobstétrique, la chirurgie réparatrice et l'ophtalmologie) (74\% de femmes contre $26 \%$ d'hommes). Les autres facteurs démographiques) n'étaient pas associés au choix de spécialité $(p>0,5)$ (tableau I).

D'autres facteurs semblaient jouer un rôle dans le choix de spécialité (tableau II), notamment la durée de la formation, le défi perçu, le lien de parenté avec un professionnel de santé, le profil personnel, la responsabilité et la nature de la spécialité. En revanche, il n'y avait pas de différence significative pour le choix de la spécialité selon la disponibilité du poste, l'affectation à la fin de la spécialité et la qualité de formation.

\section{Échelles de valeurs et choix de la spécialité}

Les échelles de valeurs avaient une bonne fiabilité interne, le coefficient alpha de Cronbach global étant de 0,80 et variant entre 0,66 pour la sous-échelle « prestige » et 0,86 pour la sous-échelle « revenu ».

Dans les six échelles de valeurs, certaines variables étaient associées significativement, dans un sens ou dans l'autre, avec les choix de carrière des étudiants. Ainsi dans l'échelle de valeur « intérêt académique », les variables «pour réaliser des recherches dans mon domaine» et «pour travailler dans un milieu universitaire » étaient associées au choix de chirurgie (avec des valeurs de $p$ respectivement de 0,012 et 0,027 ).

Dans l'échelle de valeur «prestige», les variables «parce que j'aurai l'opportunité d'effectuer de la chirurgie », « parce que je désire une carrière de spécialité que n'importe qui ne peut faire» et «pour pouvoir utiliser mes compétences techniques et manuelles » étaient fortement associées au choix de chirurgie (avec des valeurs de $p$ respectivement de $<0,001,0,013$ et 0,0001 ).

Dans l'échelle de valeur « rôle de soutien », une seule variable - « pour s'identifier à d'autres médecins qui pratiqueront dans mon domaine »-était associée au choix de chirurgie $(p=0,031)$.

Enfin, dans l'échelle de valeur «évitement des contraintes », la variable «parce que les heures de travail seront stables et prévisibles » était liée aux spécialités de médecine, en particulier la pédiatrie, la gastroentérologie, la dermatologie et la néphrologie $(p=0,014)$.

Les échelles « orientation biosociale et bioscientifique » et « revenu » n'ont montré aucune association positive avec le choix de la spécialité des médecins (tableau III).

\section{Discussion}

L'accession de plus en plus de femmes à l'enseignement supérieur constitue un facteur prépondérant de la diversification de la population étudiante ${ }^{[7-9]}$. D'après Nabli et al. ${ }^{[10]}$, les femmes valorisent plus 


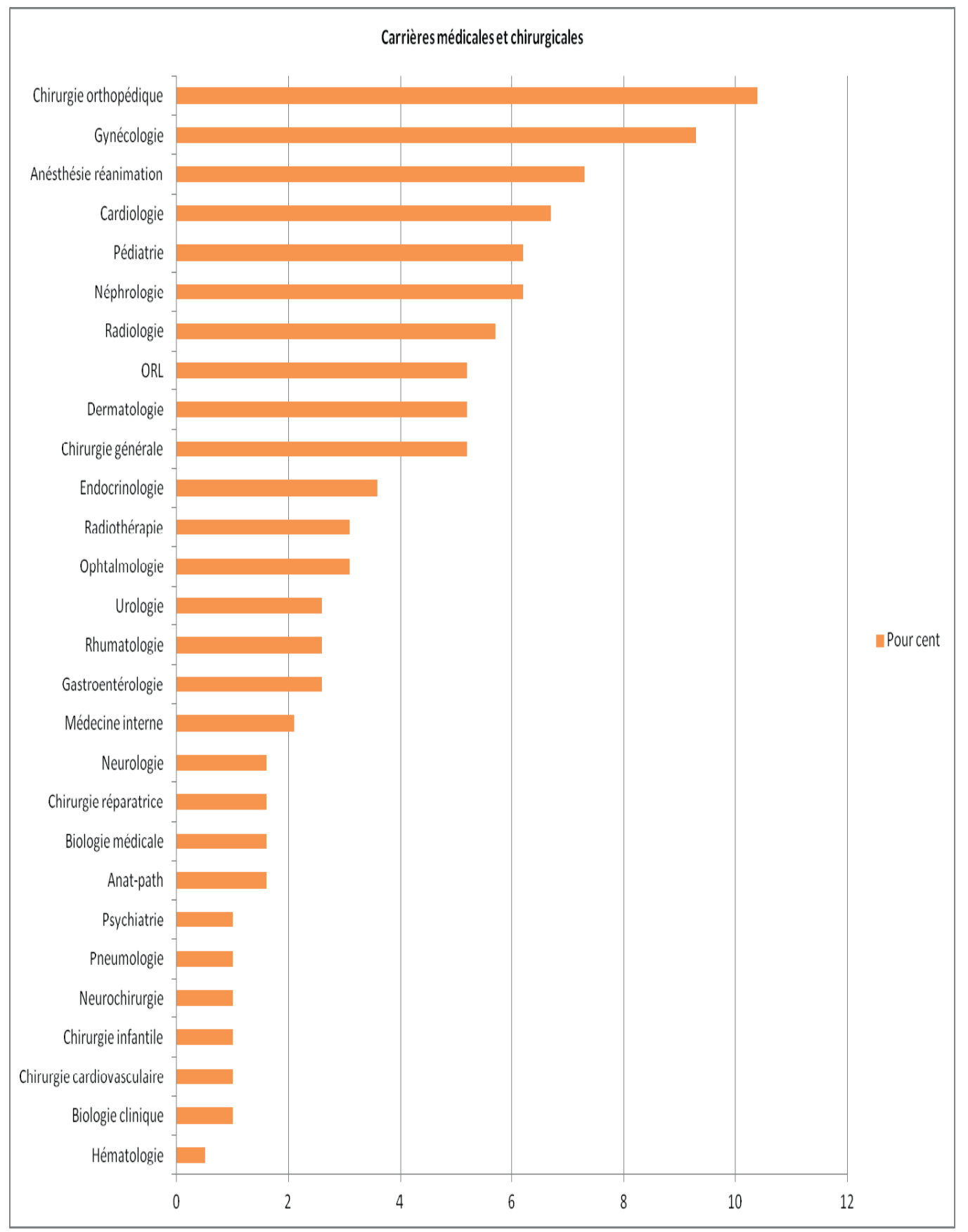

Fig. 1. Répartition des médecins résidents participant à l'étude selon la spécialité.

que les hommes les aspects intrinsèques du rôle du médecin, tels que le désir d'aider autrui et de travailler avec les gens. Elles accordent plus d'importance à la qualité de la relation avec leurs patients et démontrent plus d'empathie et une meilleure écoute envers eux ${ }^{[10,11]}$. Dans notre étude, les résidents de sexe féminin représentaient $59 \%$ des participants. On a noté également un accroissement de la proportion de 
Tableau I. Influence des facteurs sociodémographiques sur le choix de la spécialité.

\begin{tabular}{|c|c|c|c|c|c|}
\hline \multirow[t]{3}{*}{ Variable et modalités } & \multicolumn{4}{|c|}{ Spécialité } & \multirow[t]{3}{*}{$p$} \\
\hline & \multicolumn{2}{|c|}{ Médicale } & \multicolumn{2}{|c|}{ Chirurgicale } & \\
\hline & $n$ & $\%$ & $n$ & $\%$ & \\
\hline \multicolumn{5}{|l|}{ sexe } & \multirow[t]{3}{*}{$P<0,001$} \\
\hline Homme & 25 & 31,6 & 54 & 68,4 & \\
\hline Femme & 90 & 78,9 & 24 & 21,1 & \\
\hline \multicolumn{5}{|l|}{ État civil } & \multirow[t]{3}{*}{$p=0,836$} \\
\hline Marié & 37 & 32,2 & 24 & 30,8 & \\
\hline Célibataire & 78 & 67,8 & 54 & 69,2 & \\
\hline $\begin{array}{l}\text { Membre de la famille } \\
\text { professionnel de santé }\end{array}$ & 55 & 47,8 & 39 & 50,0 & $p=0,766$ \\
\hline \multicolumn{5}{|l|}{ Niveau d'étude du père } & \multirow[t]{5}{*}{$P=0,973$} \\
\hline Primaire & 14 & 12,2 & 11 & 14,1 & \\
\hline Secondaire & 21 & 18,3 & 15 & 19,2 & \\
\hline Licence & 53 & 46,1 & 34 & 43,6 & \\
\hline Master et doctorat & 27 & 23,5 & 18 & 23,1 & \\
\hline \multicolumn{5}{|l|}{ Niveau d'étude de la mère } & \multirow[t]{4}{*}{$p=0,879$} \\
\hline Primaire & 33 & 28,7 & 25 & 32,1 & \\
\hline Secondaire & 38 & 33,0 & 25 & 32,1 & \\
\hline Licence Master et doctorat & 44 & 38,3 & 28 & 35,9 & \\
\hline Travaux volontariat & 52 & 66,7 & 62 & 53,9 & $p=0,077$ \\
\hline
\end{tabular}

Tableau II. Influence des facteurs personnels sur le choix de la spécialité.

\begin{tabular}{|l|c|c|c|c|c|}
\hline \multirow{2}{*}{ Variable et modalités } & \multicolumn{3}{|c|}{ Spécialité } & \multirow{2}{*}{$\boldsymbol{p}$} \\
\cline { 2 - 6 } & \multicolumn{2}{|c|}{ Médicale } & \multicolumn{2}{c|}{ Chirurgicale } \\
\cline { 2 - 5 } & $\boldsymbol{n}$ & $\boldsymbol{\eta}$ & $\boldsymbol{n}$ & $\mathbf{\%}$ & \\
\hline Durée de la formation & 68 & 59,1 & 6 & 7,7 & $\mathbf{0 , 0 0 1}$ \\
\hline Disponibilité du poste & 49 & 42,6 & 23 & 29,5 & 0,064 \\
\hline Affectation spécialité & 24 & 20,9 & 19 & 24,4 & 0,567 \\
\hline Qualité de formation & 66 & 57,4 & 48 & 61,5 & 0,565 \\
\hline Défi & 50 & 43,5 & 55 & 70,5 & $\mathbf{0 , 0 0 0 2}$ \\
\hline Influence de la famille & 22 & 19,1 & 21 & 26,9 & 0,201 \\
\hline Lien de parenté & 11 & 9,6 & 22 & 28,2 & $\mathbf{0 , 0 0 0 7}$ \\
\hline Profil personnel & 75 & 65,2 & 39 & 50,0 & $\mathbf{0 , 0 3 4}$ \\
\hline Choc émotionnel & 8 & 7,0 & 4 & 5,1 & 0,605 \\
\hline Responsabilité & 28 & 24,3 & 42 & 53,8 & $<\mathbf{0 , 0 0 1}$ \\
\hline Nature de spécialité & 20 & 17,4 & 37 & 47,4 & $<\mathbf{0 , 0 0 1}$ \\
\hline
\end{tabular}


Tableau III. Influence des échelles de valeur sur le choix de la spécialité,

\begin{tabular}{|c|c|c|}
\hline Variable et modalités & Khi2 $\left(\chi^{2}\right)$ & $p$ \\
\hline \multicolumn{3}{|l|}{ Facteur 1 : Orientation biosociale et bioscientifique } \\
\hline Développer des rapports à long terme & 0,79 ns à $3 \mathrm{ddl}$ & $p=0,850$ \\
\hline Se concentrer sur les besoins sociaux & 2,67 ns à 3 ddl & $p=0,445$ \\
\hline Traiter et suivre les familles en consultation & 5,64 ns à 3 ddl & $p=0,130$ \\
\hline Pratiquer la médecine préventive & $2,85 \mathrm{~ns}$ à $4 \mathrm{ddl}$ & $p=0,583$ \\
\hline \multicolumn{3}{|l|}{ Facteur 2 : Intérêt académique } \\
\hline Réaliser des recherches dans mon domaine & 12,74 à 4 ddl & $p=0,012$ \\
\hline Découvrir de nouveau traitement ou thérapie & 2,96 ns à 4 ddl & $p=0,564$ \\
\hline Travailler dans un milieu universitaire & 10,88 à 4 ddl & $p=\mathbf{0 , 0 2 7}$ \\
\hline \multicolumn{3}{|l|}{ Facteur 3 : Prestige } \\
\hline Avoir l'opportunité d'effectuer la chirurgie & 115,67 à 2 ddl & $p<0,001$ \\
\hline Carrière de spécialité que n'importe qui ne peut faire & 12,59 à 4 ddl & $p=\mathbf{0 , 0 1 3}$ \\
\hline Utiliser les compétences techniques et manuelles & 20,12 à 3 ddl & $p=0,0001$ \\
\hline Utiliser les dernières avancées technologiques & 8,95 ns à 4 ddl & $p=0,062$ \\
\hline \multicolumn{3}{|l|}{ Facteur $4:$ Revenu } \\
\hline Carrière bien rémunérée & 2,59 ns à 4 ddl & $p=0,628$ \\
\hline Carrière assure un bon revenu pour acheter une belle maison & $6,1 \mathrm{~ns}$ à $3 \mathrm{ddl}$ & $p=0,108$ \\
\hline Carrière assure un bon revenu pour faire des vacances exotiques & 4,7 ns à 3 ddl & $p=0,195$ \\
\hline \multicolumn{3}{|l|}{ Facteur 5 : Évitement des contraintes } \\
\hline Les heures de travail seront à temps partiel & 7,6 à 3 ddl & $p=0,054$ \\
\hline Les heures de travail seront stables et prévisibles & 10,56 à 3 ddl & $p=0,014$ \\
\hline Je peux partager mon poste avec d'autres médecins & 5,56 ns à 3 ddl & $p=0,135$ \\
\hline \multicolumn{3}{|l|}{ Facteur 6 : Rôle de soutien } \\
\hline Je vais travailler avec des personnes ayant des valeurs & 6,34 ns à 4 ddl & $p=0,175$ \\
\hline Je ne serai pas dérangé étant la seule personne dans mon domaine & 2,03 ns à 3 ddl & $p=0,567$ \\
\hline S'identifier à d'autres médecins qui pratiquent dans mon domaine & 8,86 à 3 ddl & $p=\mathbf{0 , 0 3 1}$ \\
\hline
\end{tabular}

ns : non significatif, ddl : degré de liberté.

femmes dans le cursus de résidanat puisqu'elle est passée de 16,6\% à 71,4\% en cinq ans.

Parmi les facteurs démographiques, le sexe était significativement associé $(p<0,001)$, en analyse univariée, avec le choix de carrière et avec les échelles de valeurs. Par contre, les travaux de volontariat, le niveau d'éducation des parents, le classement au concours et l'âge ne prédisaient pas le choix de spécialité d'un étudiant, ce qui confirme les résultats d'autres études ${ }^{[6,12]}$.

Selon le modèle de Murdoch ${ }^{[5]}$, dans les échelles de valeurs « intérêt académique, prestige, rôle de soutien », plusieurs variables sont liés aux choix plus «masculins » de chirurgie et de spécialisations académiques, tandis que dans l'échelle « évitement des contraintes », une variable était plus « féminine » et liée aux spécialités médicales ainsi qu'aux professions de soins primaires.

Les femmes présentent plus de réticences envers les contraintes professionnelles en rapport, entre autres, avec les défis de la maternité et leur responsabilité familiale ${ }^{[10,13,14]}$. Dans notre étude portant sur 193 étudiants, elles accordent plus de poids que les hommes à des caractéristiques de carrière du groupe 
« évitement des contraintes », comme un emploi du temps malléable et des facilités au lieu de travail $(p=<0,05)$. Les femmes abordent et mènent leur carrière de façon distincte de celle des hommes. Étant très sensibles aux conditions de travail qui doivent leur permettre de concilier vie professionnelle et vie familiale, les femmes semblent plus souvent moduler leur carrière en fonction de considérations familiales ${ }^{[15,16]}$.

Cependant, notre étude présente quelques limites méthodologiques. Le taux de non-répondants était de $26,4 \%$; on en ignore le profil et l'impact potentiel sur les résultats. L'échantillon de médecins interrogés était faible et notre étude n'a porté que sur une seule faculté de médecine. Cependant, il s'agissait d'un travail d'évaluation préliminaire ; une analyse multi-institutionnelle incluant les autres facultés de médecine et de pharmacie (Casablanca, Rabat et Fès) permettra de tirer des conclusions valides à l'échelle nationale. Une étude plus large permettrait également de se comparer au pays du Maghreb et d'expliquer d'éventuelles variations d'un pays à l'autre. Nous n'avons utilisé qu' un seul outil d'évaluation (le questionnaire). C'est une méthode qui a pour avantage d'être simple, facile à exploiter et anonyme. Ses limites sont d'être une technique fermée et non interactive, ce qui peut limiter la discussion. Ultérieurement, l'utilisation d'autres outils d'évaluation devrait nous permettre de mieux identifier et d'évaluer d'autres facteurs exprimés et ressentis.

\section{Conclusion}

Le sexe, des facteurs personnels et les échelles de valeurs (intérêt académique, prestige, rôle de soutien, évitement des contraintes) ; sont les principaux facteurs qui influencent le choix de carrière des médecins résidents. Or, le caractère classant du concours de résidanat pourrait induire d'autres déterminants du choix des futures filières de spécialités, car il est basé sur des épreuves jugeant les performances académiques et conduit à un classement par ordre de mérite, obligeant certains étudiants à des choix très restreints ne correspondant pas à leurs souhaits.

\section{Contributions}

Aboubakr Matrane a conçu le protocole d'étude, participé à la collecte des données et à l'interprétation et rédigé l'article. Zineb Serhier et Mohammed Bennani Othmani ont effectué l'analyse statistique, participé à l'interprétation des résultats et à la révision critique du manuscrit.

\section{Orientation biosociale et bioscientifique (Bioso- cial and Bioscientific Orientation)}

Traiter le patient comme un tout fonctionnel, en tant qu'individu, avec les implications psychosociales que cela entend.

Désir d'épanouissement intellectuel et de familiarisation avec les techniques de pointe.

\section{Intérêt académique (Academic interest)}

Travail dans un contexte académique propice aux travaux de recherche et désir d'enseigner.

\section{Prestige (Prestige)}

Reconnaissance sociale de ses capacités et de ses « exploits ».

\section{Revenu (Income)}

Attente d'un revenu élevé et d'une vie aisée en carrière postdoctorale.

\section{5. Évitement des contraintes (Avoid role strain)}

Travailler sans contraintes, à son rythme et garder une souplesse d'emploi du temps compatible avec la vie de famille.

\section{Rôle de soutien (Role support)}

Ensemble de caractéristiques démographiques et personnelles qui rendent le résidanat et la profession plus 
« rassurants » pour certains candidats, anxieux à l'idée de travailler en milieu inconnu (socialement).

\section{Références}

1. Décret $n^{\circ} 2-91-527$ relatif à la situation des externes, des internes et des résidants des centres hospitaliers. 1993 [On-line] Disponible sur : http:// srvweb.sante.gov.ma/Reglementation/exinre/ Documents/Décret n²-91-527.pdf. 1.

2. Plan d'action santé, 2008-2012 «Réconcilier le citoyen avec son système de santé ». [On-line] Disponible sur: http://www.sante.gov.ma/INAS/pdf/ Plan-action-08-12.pdf.

3. Sbai Idrissi K. La santé dans le royaume du Maroc. Med Trop 2002;62:489-96.

4. Anthioumane, Tandia. Système de santé : où va le Maroc ? Conjoncture 2008;890:21-30.

5. Murdoch MM, Kressin N, Fortier L, Giuffre PA, Oswald L. Evaluating the psychometric properties of a scale to measure medical students' career-related values. Acad Med 2001;76:157-65.

6. Choucair J, Nemr E, Sleillaty G, Abboud M. Choix de la spécialité en médecine : Quels facteurs influencent la décision des étudiants ? Pédagogie Médicale 2007;8:145-55.

7. Lapeyre N, Le Feuvre N. Féminisation du corps médical et dynamiques professionnelles dans le champ de la santé. Revue Française des Affaires sociales 2005;1:59-81.

8. Hamel MB, Ingelfinger JR, Phimister E, Solomon C. Women in Academic Medicine - Progress and Challenges. New Engl J Med 2006;355:310-2.

9. Magrane D, Lang J. An overview of women in U.S. academic medicine, 2005-2006. Analysis in Brief,
Volume 6, Number 7. Washington, DC: Association of American Medical Colleges; 2006. [On-line] Disponible sur: http://www.aamc.org/data/aib/ aibissues/aibvol6_no7.pdf.

10. Nabli Ajmi T, Bougmiza MI, Mtiraoui A. Attitudes des étudiants tunisiens en médecine vis-à-vis de la pratique médicale : différences en fonction du sexe. East Mediterr Health J 2008;14:686-96.

11. Chouinard A, Chouinard R. Les facteurs d'influence de la participation verbale en classe des étudiants universitaires: une revue de la littérature. Revue internationale de pédagogie de l'enseignement supérieur [en ligne]. 2009. [On-line] Disponible sur: http://ripes.revues.org/59.

12. Owen JA, Hayden GF, Connors AF Jr. Can medical school admission committee members predict which applicants will choose primary care careers? Acad Med 2002;77:344-9.

13. Mobilos S, Chan M, Brown JB. Women in medicine: the challenge of finding balance. Can Fam Physician 2008;54:1285-86.e5.

14. Serrano K. Women residents, women physicians and medicine's future. WMJ 2007;106:260-5.

15. Buddeberg-Fischer B-F, Klaghofer R, Abel T, Buddeberg $\mathrm{C}$. The influence of gender and personality traits on the career planning of Swiss medical students. Swiss Med Wkly 2003;133:535-40.

16. Rombouts J-J, Godin V. La féminisation de la profession médicale : la carrière académique et hospitalière des femmes médecins. Louvain Médical 2004; 123:338-41.

Correspondance et offprints: Aboubakr Matrane, Service Médecine Nucléaire, CHU Mohammed VI, Marrakech, Maroc. Mailto: matrane33@yahoo.fr. 


\section{Le questionnaire}

\section{$\underline{\text { I, Caractéristiques sociodémographiques }}$}

1. Sexe: $\mathrm{F} \quad \mathrm{M}$

2. Âge : ans

3. Année de résidanat : $\quad 1^{\mathrm{ère}}$ année $\quad 2^{\mathrm{e}}$ année $\quad 3^{\mathrm{e}}$ année

4. État civil : Célibataire Marié Divorcé

5. Est-ce que vous avez un membre de famille qui est professionnel de santé ?

\section{Oui Non}

Si oui, quel est le lien de parenté ? Père Mère Autre

Précisez sa profession : Père :

Mère :

Autre:

6. Niveau d'éducation du père : Primaire Secondaire

Licence

7. Niveau d'éducation de la mère: $\begin{aligned} & \text { Master } \\ & \text { Primaire }\end{aligned}$

Doctorat

Master Doctorat

Licence

8. Travaux de volontariat

Avez-vous déjà fait du bénévolat ? Oui

Non

\section{II, Choix de la spécialité}

9. Est-ce que vous êtes :

10. Quelle est votre spécialité :

11. Vous êtes :

Résident sur concours

Résident sur titre

Bénévole

12. Quel était votre classement au concours (résidanat ou internat) :

Parmi les 10 premiers

Au milieu

les 10 derniers

\section{III, Facteurs influencant le choix de la spécialité}

Quels sont les facteurs qui vous ont poussé à choisir votre spécialité ?

Durée de formation

Disponibilité du poste

Affectation à la fin de la spécialité

Qualité de la formation

Défi

Culte et tradition

Influence de la famille (ou conjoint)

Profil personnel

Choc émotionnel (décès ou maladie grave d'un proche)

Responsabilité

Nature de la spécialité médico-chirurgicale

Autre, précisez 


\section{$\underline{\text { IV- Quelle était l'influence des items suivants sur votre choix de spécialité }}$}

\begin{tabular}{|c|c|c|c|c|c|}
\hline $\begin{array}{l}\text { Les Raisons de mon choix } \\
\text { de spécialité }\end{array}$ & $\begin{array}{c}\text { pas } \\
\text { d'influence }\end{array}$ & $\begin{array}{c}\text { peu } \\
\text { d'influence }\end{array}$ & $\begin{array}{l}\text { influence } \\
\text { moyenne }\end{array}$ & $\begin{array}{l}\text { influence } \\
\text { importante }\end{array}$ & $\begin{array}{c}\text { influence } \\
\text { trés } \\
\text { importante }\end{array}$ \\
\hline \multicolumn{6}{|l|}{$\begin{array}{l}\text { Développer des rapports à long } \\
\text { terme avec mes patients }\end{array}$} \\
\hline \multicolumn{6}{|l|}{$\begin{array}{l}\text { Se concentrer sur les besoins } \\
\text { sociaux et psychologiques du } \\
\text { patient }\end{array}$} \\
\hline \multicolumn{6}{|l|}{$\begin{array}{l}\text { Traiter et suivre les familles en } \\
\text { consultation }\end{array}$} \\
\hline \multicolumn{6}{|l|}{ Pratiquer la médecine préventive } \\
\hline \multicolumn{6}{|l|}{$\begin{array}{l}\text { Réaliser des recherches dans } \\
\text { mon domaine }\end{array}$} \\
\hline \multicolumn{6}{|l|}{$\begin{array}{l}\text { Découvrir de nouveaux } \\
\text { traitements ou thérapie }\end{array}$} \\
\hline \multicolumn{6}{|l|}{$\begin{array}{l}\text { Travailler dans un milieu } \\
\text { universitaire }\end{array}$} \\
\hline \multicolumn{6}{|l|}{$\begin{array}{l}\text { Avoir l'opportunité d'effectuer } \\
\text { de la chirurgie }\end{array}$} \\
\hline \multicolumn{6}{|l|}{$\begin{array}{l}\text { Désirer une carrière de spécialité } \\
\text { que n'importe qui ne peut faire }\end{array}$} \\
\hline \multicolumn{6}{|l|}{$\begin{array}{l}\text { Utiliser mes compétences } \\
\text { (techniques et manuelle) }\end{array}$} \\
\hline \multicolumn{6}{|l|}{$\begin{array}{l}\text { Utiliser les dernières avancées } \\
\text { technologiques }\end{array}$} \\
\hline \multicolumn{6}{|l|}{$\begin{array}{l}\text { La carrière est très bien } \\
\text { rémunérée }\end{array}$} \\
\hline \multicolumn{6}{|l|}{$\begin{array}{l}\text { La carrière assure un revenu } \\
\text { suffisant pour pouvoir acheter } \\
\text { une belle maison }\end{array}$} \\
\hline \multicolumn{6}{|l|}{$\begin{array}{l}\text { La carrière assure un bon revenu } \\
\text { pour pouvoir faire des vacances } \\
\text { exotiques }\end{array}$} \\
\hline \multicolumn{6}{|l|}{$\begin{array}{l}\text { Les heures de travail seront à } \\
\text { temps partiel et flexibles }\end{array}$} \\
\hline \multicolumn{6}{|l|}{$\begin{array}{l}\text { Les heures de travail seront } \\
\text { stables et prévisibles }\end{array}$} \\
\hline \multicolumn{6}{|l|}{$\begin{array}{l}\text { Pouvoir partager mon poste de } \\
\text { travail avec d'autre médecin }\end{array}$} \\
\hline \multicolumn{6}{|l|}{$\begin{array}{l}\text { Travailler avec des personnes } \\
\text { ayant des valeurs et de l'estime }\end{array}$} \\
\hline \multicolumn{6}{|l|}{$\begin{array}{l}\text { Je ne serai pas dérangé étant la } \\
\text { seule personne dans mon } \\
\text { domaine }\end{array}$} \\
\hline $\begin{array}{l}\text { S'identifier à d'autres médecins } \\
\text { qui pratiqueront dans mon } \\
\text { domaine }\end{array}$ & & & & & \\
\hline
\end{tabular}

